MS102.001

\title{
Halogenation as a new tool to control peptide self-assembly
}

Pierangelo Metrangolo ${ }^{1}$, Nicola Demitri ${ }^{2}$, Alessandro Gori ${ }^{3}$, Andrea Pizzi ${ }^{1}$, Giancarlo Terraneo ${ }^{1}$

${ }^{1}$ Dept. Chem., Mater., And Chem. Eng. 'Giulio Natta', Politecnico Di Milano, Milano, Italy, ${ }^{2}$ Elettra-Synchrotron Light Source, Trieste, Italy, ${ }^{3}$ Istituto di Chimica del Riconoscimento Molecolare, CNR, Milano, Italy

E-mail: pierangelo.metrangolo@polimi.it

Although many modifications of peptide sequences have been utilized to tune their self-assembly, halogenation has rarely been pursued. The advantage of a strategy based on the introduction of halogen atoms on peptide motifs lies in the fact that halogenation is a minimal structural modification, which, on the other hand, may induce a large difference in the peptide supramolecular behavior as a consequence of the rich variety of noncovalent interactions given by halogen atoms [1].

In this presentation, it will be shown how halogenation strongly influences both solution and solid-state self-assembly behavior of amyloidogenic peptides. We have applied this new supramolecular concept to the augmented fibrillation of amyloidogenic peptides and proteins, such DFNKF [2], KLVFF, and hCT. Furthermore, halogenation facilitated obtaining highquality single crystals of fibril-forming peptides. In particular, iodination of the widely studied amyloidogenic peptide sequence DFNKF facilitated crystallization and allowed for its high-resolution single crystal structure determination for the first time. The structure unveils the importance of aromatic-aromatic interactions in stabilizing the amyloid self-assembly (Figure) [3]. Implications of oxidative stress-induced halogenation of proteins are discussed in terms of biomarkers of diseases such as Parkinson and Alzheimer's. The obtainment of a novel unnatural amino acid functioning as strong halogen-bond donor may pave the way to totally new design principles in peptide-based supramolecular self-assembly.

Acknowledgement: P.M. gratefully acknowledges the European Research Council (ERC) for funding the project "Folding with Halogen Bonding" to PM (FoldHalo, www.foldhalo.eu; Grant agreement no. 307108).

[1] Pizzi, A. et al. (2017). CrystEngComm 19, DOI: 10.1039/C7CE00031F.

[2] Bertolani, A. et al. (2015). Nat. Commun. 6:7574, DOI: $10.1038 /$ ncomms 8574.

[3] Bertolani, A. et al. (2017). Chem. Eur. J. 22, 2051-2058.

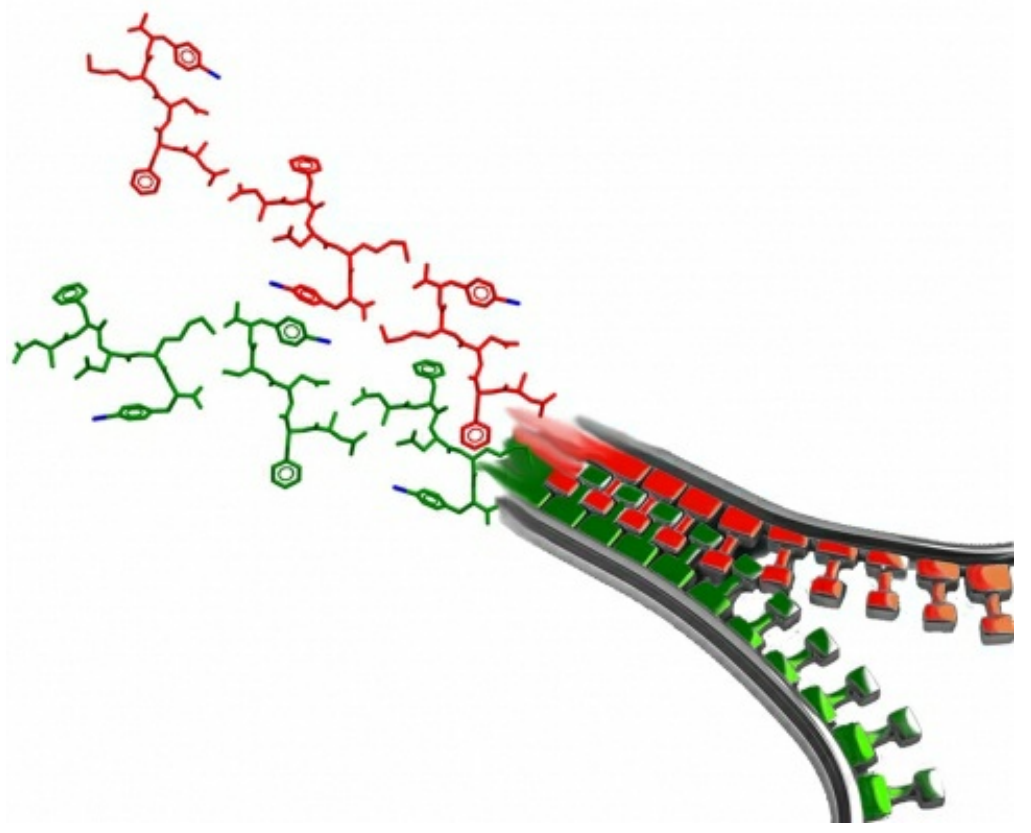

Keywords: halogenation, peptide, self-assembly 